\title{
THE AFFERENT AND EFFERENT CALLOSAL CONNECTIONS OF RETINOTOPICALLY DEFINED AREAS IN CAT CORTEX ${ }^{1}$
}

\author{
MARK A. SEGRAVES ${ }^{2}$ AND ALAN C. ROSENQUIST ${ }^{3}$ \\ Department of Anatomy, University of Pennsylvania, Philadelphia, Pennsylvania 19104 \\ Received August 18, 1981; Revised January 25, 1982; Accepted February 26, 1982
}

\begin{abstract}
We compared the callosal afferent and efferent connections of different retinotopic loci within a given visual cortical area as well as the connectivity patterns among similar retinotopic loci in different visual areas. Small injections $(75 \mathrm{nl})$ of a mixture of horseradish peroxidase and $\left[{ }^{3} \mathrm{H}\right]$ leucine were made through a recording pipette at injection sites identified by retinotopic mapping. A small locus of cortex within a callosally connected region had precise reciprocal connections with the homotopic locus in the contralateral hemisphere. This small locus also was callosally connected with a variable number of heterotopic loci. Both reciprocal and nonreciprocal heterotopic callosal connections were found. Homotopic and heterotopic connections appeared to have a high degree of retinotopic fidelity. Precisely homotopic connections were present not only between locations on the vertical meridian representations at the left and right area 17/18 borders but also, for example, between mirror-symmetrical points on the peripheral horizontal meridian representation in the left and right posteromedial lateral suprasylvian areas. In several experiments, we found that both callosal neurons and terminals in the homotopic cortex were grouped into two to three distinct clusters ranging from 600 to $900 \mu \mathrm{m}$ in width. Callosal neurons with homotopic connections were primarily pyramidal cells in lower layer III and upper layer IV. Outside of areas 17 and 18, there was a significant number of pyramidal and fusiform-shaped callosal neurons in layers V and VI. The majority of callosal terminals were located in layers II, III, and IV.
\end{abstract}

The preceding paper (Segraves and Rosenquist, 1982) described the distribution of cells that project through the corpus callosum in each of the 13 retinotopically defined areas of cat visual cortex. In this paper, we compare the callosal afferent and efferent connections of different retinotopic loci within a given visual area, and we compare connectivity patterns among similar retinotopic loci of different visual areas. This information is important for a comprehensive understanding of the overall callosal connectivity and function in vision as well

\footnotetext{
'This work was supported by National Institutes of Health Grants EY02654 and GM00281 and by a Grass Foundation fellowship award to M. A. S. We wish to thank Jeanne Levy for technical assistance as well as John and Bette Woolsey, Tom Sarrantonio, and Pauline Wong for preparation of the tables and figures. Denis Raczkowski, Elin F. S. Kaufman, and Laura Symonds participated in many of the experiments described in this paper. We have presented preliminary reports of this study elsewhere (Segraves, 1979; Segraves and Rosenquist, 1980).

${ }^{2}$ Present address: Institute of Anatomy, University of Lausanne, Rue du Bugnon 9, 1011 Lausanne CHUV, Switzerland.

${ }^{3}$ To whom correspondence should be addressed at Department of Anatomy, University of Pennsylvania, 36th and Hamilton Walk, Philadelphia, PA 19104.
}

as for a greater appreciation of the different roles that cach visual area may play in mediating these functions.

\section{Materials and Methods}

Each of 17 cats was prepared for electrophysiological recording following procedures similar to those employed by Tusa et al. (1978). We mapped receptive field positions in the cortical region of interest and defined enough corlical retinotopic landmarks to locate the intended injection site. We then replaced the recording electrode (either an insulated tungsten electrode or a $0.75 \mathrm{M} \mathrm{NaCl}-$ filled pipette) with a pipette (10- to $15-\mu \mathrm{m}$ tip) filled with $15 \%$ horseradish peroxidase (HRP) and $\left[{ }^{3} \mathrm{H}\right]$ leucine (50 to $150 \mu \mathrm{Ci} / \mu \mathrm{l})$. The $\mathrm{HRP}$ and $\left[{ }^{3} \mathrm{H}\right]$ leucine were dissolved in Tris-buffered $0.75 \mathrm{M} \mathrm{NaCl}, \mathrm{pH} 8.6$ (Sakai et al., 1978). Preliminary experiments were used to determine the concentrations of HRP and $\left[{ }^{3} \mathrm{H}\right] l$ leucine that would produce injections of approximately equal dimensions. During an injection penetration, we recorded multiple unit neural activity to verify the receptive field center of the injection site and then injected approximately $75 \mathrm{nl}$ of the HRP $/\left[{ }^{3} \mathrm{H}\right]$ leucine mixture. Injection was by pressure introduced to the pipette-holding chamber via a solenoidoperated valve. A calibrated micrometer reticle in the 
operating microscope enabled us to measure the amount of $\mathrm{HRP} /\left[{ }^{3} \mathrm{H}\right]$ leucine mixture injected. Infusion of paralyzing agents was stopped at the time of injection, and the cat was maintained on the respirator until it was capable of breathing on its own. Following a 48-hr survival period, the cat was anesthetized deeply with sodium pentobarbital and perfused with saline, followed by a solution of $0.5 \%$ paraformaldehyde and $2.5 \%$ glutaraldehyde in phosphate buffer ( $\mathrm{pH} 7.2$ ), and finally with a solution of $5 \%$ sucrose in phosphate buffer. The brain was blocked, removed from the cranium, and left overnight in $30 \%$ sucrose in water. Forty-eight-micrometer frozen sections were cut in the coronal plane. One series of sections, at $288-\mu \mathrm{m}$ intervals, was stained for HRP

TABLE I

Summary of data from combined $H R P /\left[{ }^{3} H\right] l e u c i n e$ injection experiments

The injection receptive field center is written in the form: azimuth, elevation. The presence of HRP or $\left[{ }^{3} \mathrm{H}\right]$ leucine label in an area is indicated by a black box. A question mark signifies that the presence of label in an area was uncertain because it was near a border or because the border was difficult to define. No HRP-labeled neurons were found in case $\mathrm{AH}-24$, and no $\left[{ }^{3} \mathrm{H}\right]$ leucine-labeled terminals were found in cases $\mathrm{AH}-24, \mathrm{AH}-20$, and $\mathrm{AH}-3$. The names of the anteromedial (AMLS), posteromedial (PMLS), ventral (VLS), anterolateral (ALLS), posterolateral (PLLS), and dorsal lateral suprasylvian (DLS) areas of Palmer et al. (1978) are used.

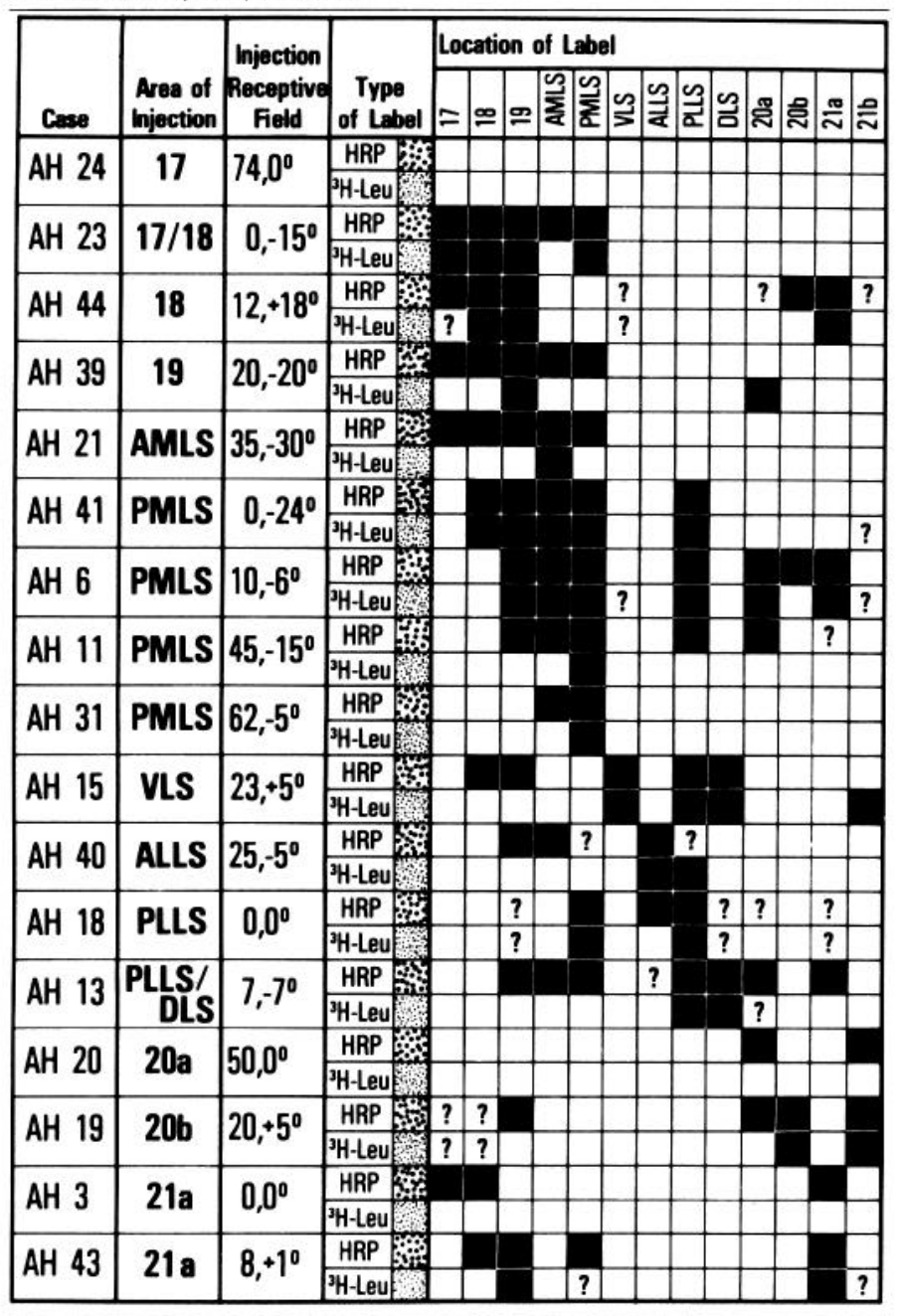
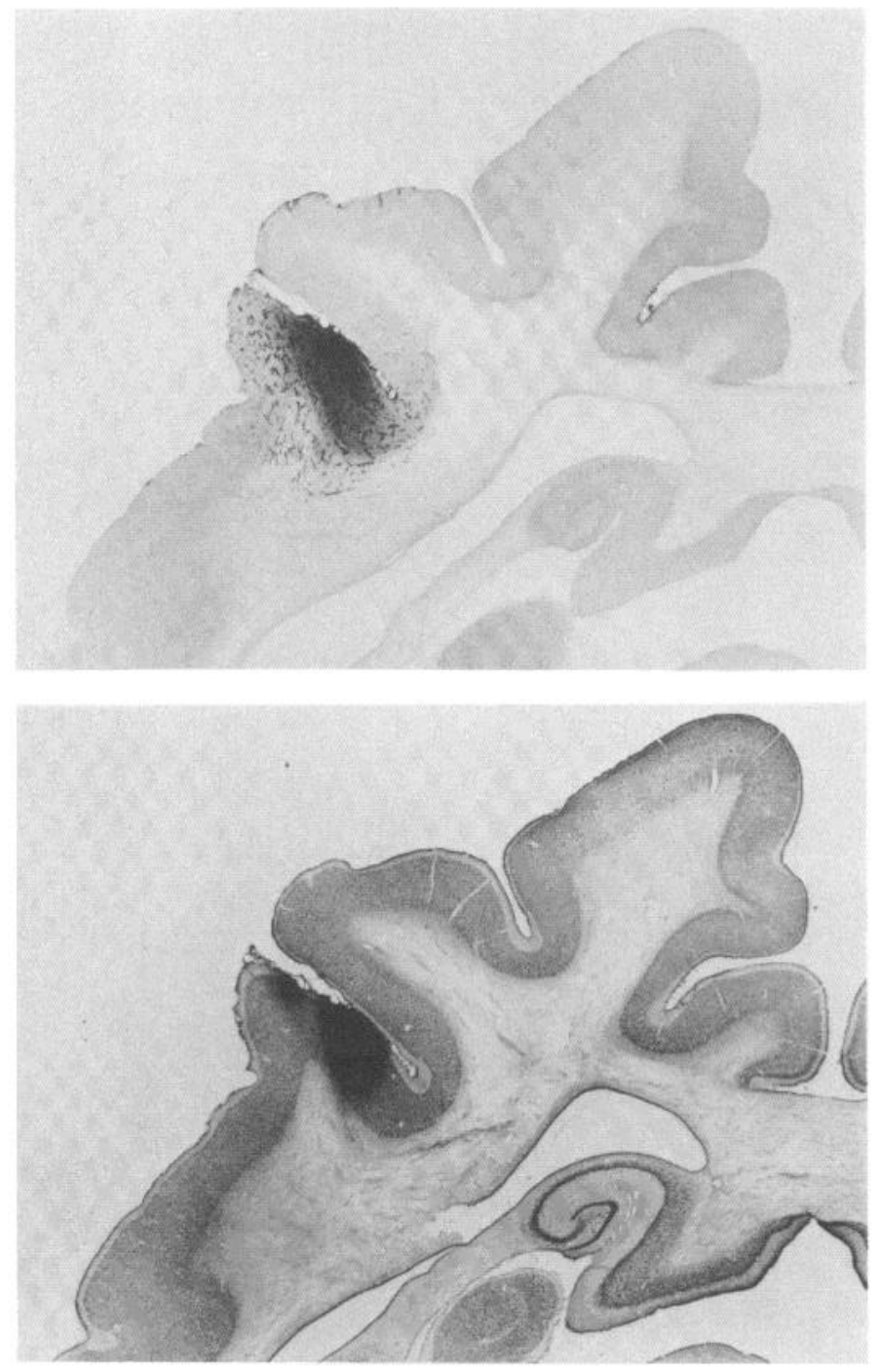

Figure 1. HRP- (above) and $\left[{ }^{3} \mathrm{H}\right]$ leucine- (below) processed sections through an injection site in area PLLS (experiment AH-18). The same case is illustrated in Figure 7. Seventy-five nanoliters of a mixture of HRP and $\left[{ }^{3} \mathrm{H}\right]$ leucine was injected from a recording pipette. The receptive field of multiple unit activity at the injection site was $0,0^{\circ}$. The HRP section was reacted with $o$-dianisidine but was not counterstained. The $\left[{ }^{3} \mathrm{H}\right]$ leucine section was counterstained with thionin.

using o-dianisidine (De Olmos, 1977) and coverslipped without counterstaining. A second series was stained for HRP and counterstained with thionin. A third series of sections, processed for autoradiography, was mounted on glass slides, dipped in Kodak NTB-2 emulsion, exposed for 8 to 16 weeks, developed, and counterstained with thionin (Cowan et al., 1972). Where necessary for identification of myeloarchitectonic borders, additional sections were stained for myelin using a Heidenhain protocol. Our methods for defining borders between visual areas were identical to those described in the preceding report (Segraves and Rosenquist, 1982).

This paper includes the results of single injections of combined HRP and $\left[{ }^{3} \mathrm{H}\right]$ leucine injections in 17 cats (Table I; Fig. 1). All injections were made in the left hemisphere, and injection sites were located in each of 

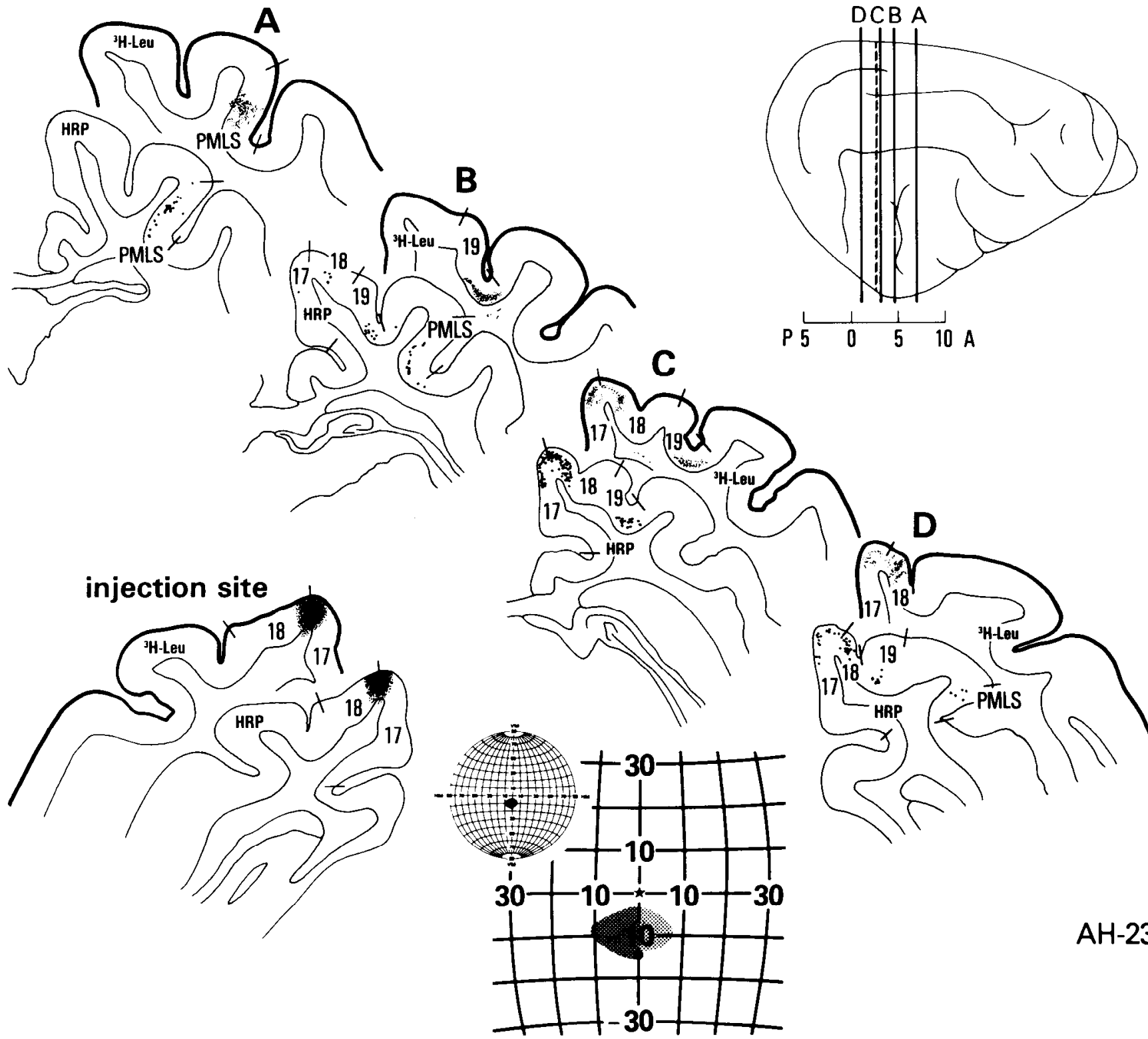

C

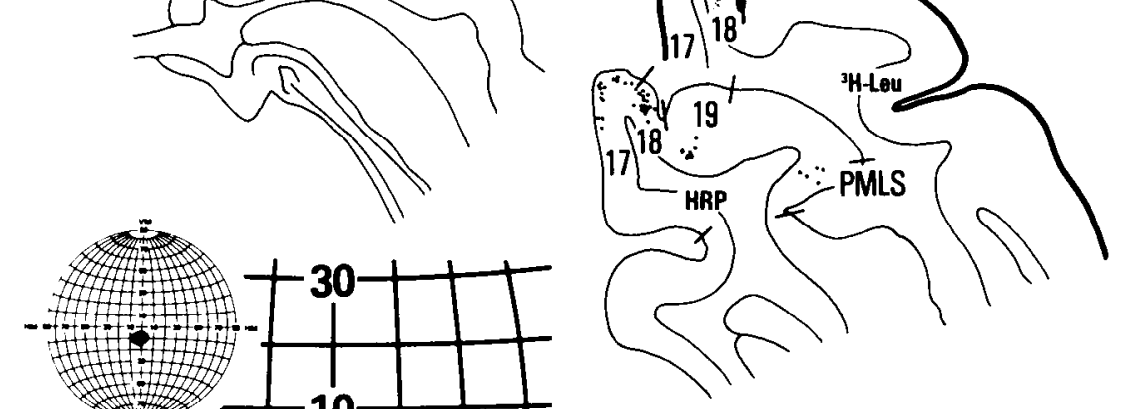

Figure 2. Injection at the area $17 / 18$ border. The receptive field center of the injection site was 0, -15 (case AH-23). Each of Figures 2 to 7 includes a series of adjacent HRP- and $\left[{ }^{3} \mathrm{H}\right]$ leucine-labeled sections showing the distribution of labeled neurons and terminals as well as the size and position of the injection. The blackened region of the HRP injection represents the very intensely stained region at the center of the injection where few neurons are visible. The extent of this region was used to estimate the area of the visual field representation encompassed by the injection. The stippling represents the more lightly stained area at the perimeter of the injection where many HRP-filled neurons are visible. A surface drawing of the right hemisphere shows the anterior-posterior levels of the sections included in the figure as well as the level of the injection site in the left hemisphere (dashed line). The Globe (bottom center) represents left and right visual hemifields. In this figure, an enlargement of the globe of $30^{\circ}$ radius from the area centralis is included. The receptive field center of multiple unit activity recorded at the injection site is indicated by a black dot. We compared the injection site with standard electrophysiological maps (Tusa et al., 1981) and estimated the area of the visual field representation encompassed by the injection. This area is shaded in the right hemifield of the globe. In the left hemifield of the globe, shading covers an estimate of the area of visual field representation in the contralateral homotopic cortex that contains labeled cells. HM, Horizontal meridian; VM, vertical meridian.

13 retinotopically defined visual areas except area $21 \mathrm{~b}$. The results of injections at the area $17 / 18$ border and in areas 19,20a, and 21a as well as in the posteromedial and posterolateral lateral suprasylvian areas are illustrated in Figures 2 to 7.

Equivalent cortical areas in the left and right hemi- spheres will be referred to as homotopic cortical areas. Projections between equivalent areas, for example from area 19 in the left hemisphere to area 19 in the right hemisphere, will be called homotopic connections. Nonequivalent visual areas, for example left area 17 and the right posteromedial lateral suprasylvian area, will be 

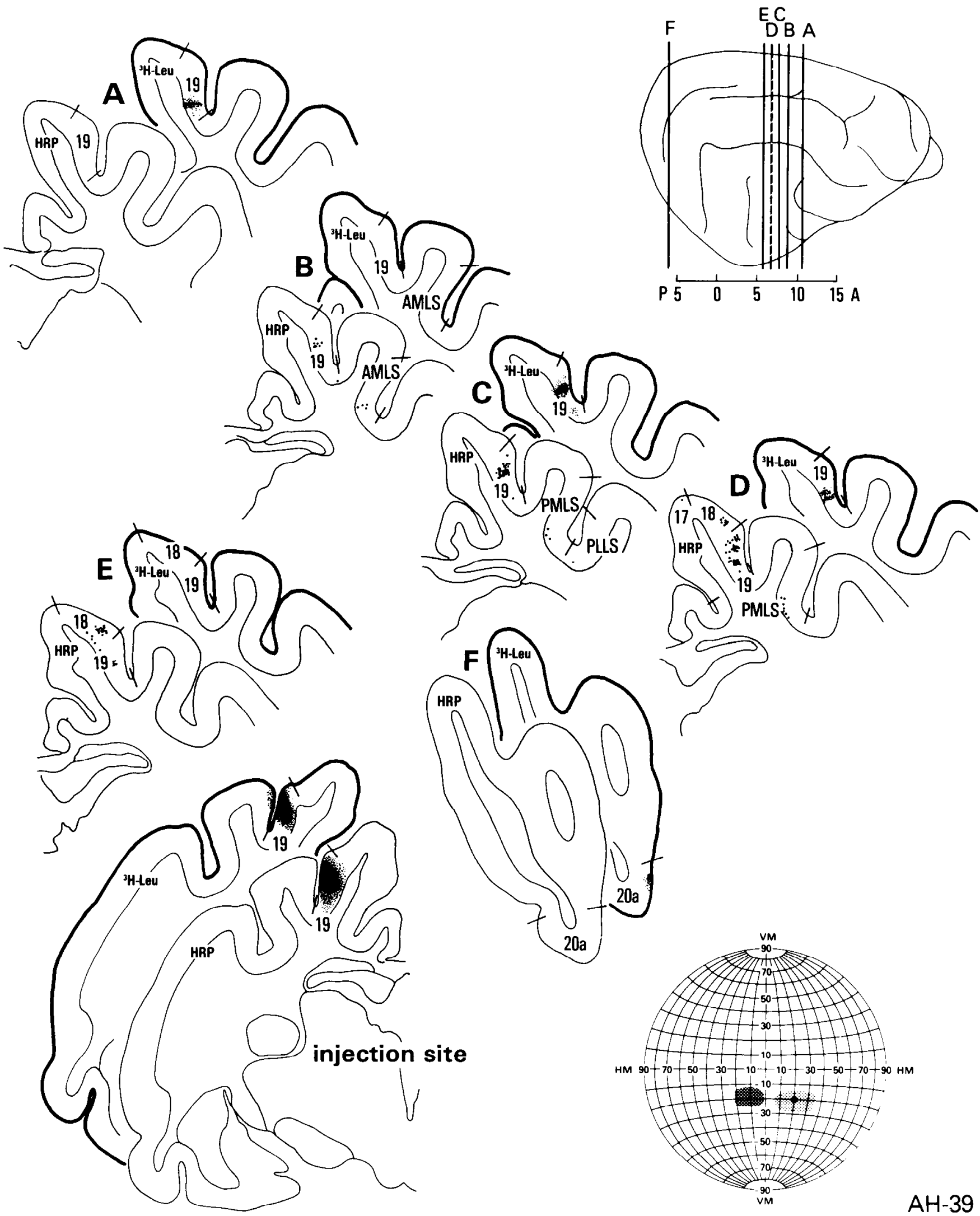

Figure 3. Injection in area 19 . The receptive field center of the injection site was $20,-20^{\circ}$ (case AH-39). The format of this figure is same as that of Figure 2. The HRP sections at levels $A$ and $F$ and $\left[{ }^{3} \mathrm{H}\right]$ leucine sections at levels $B$ and $E$ did not contain label. 

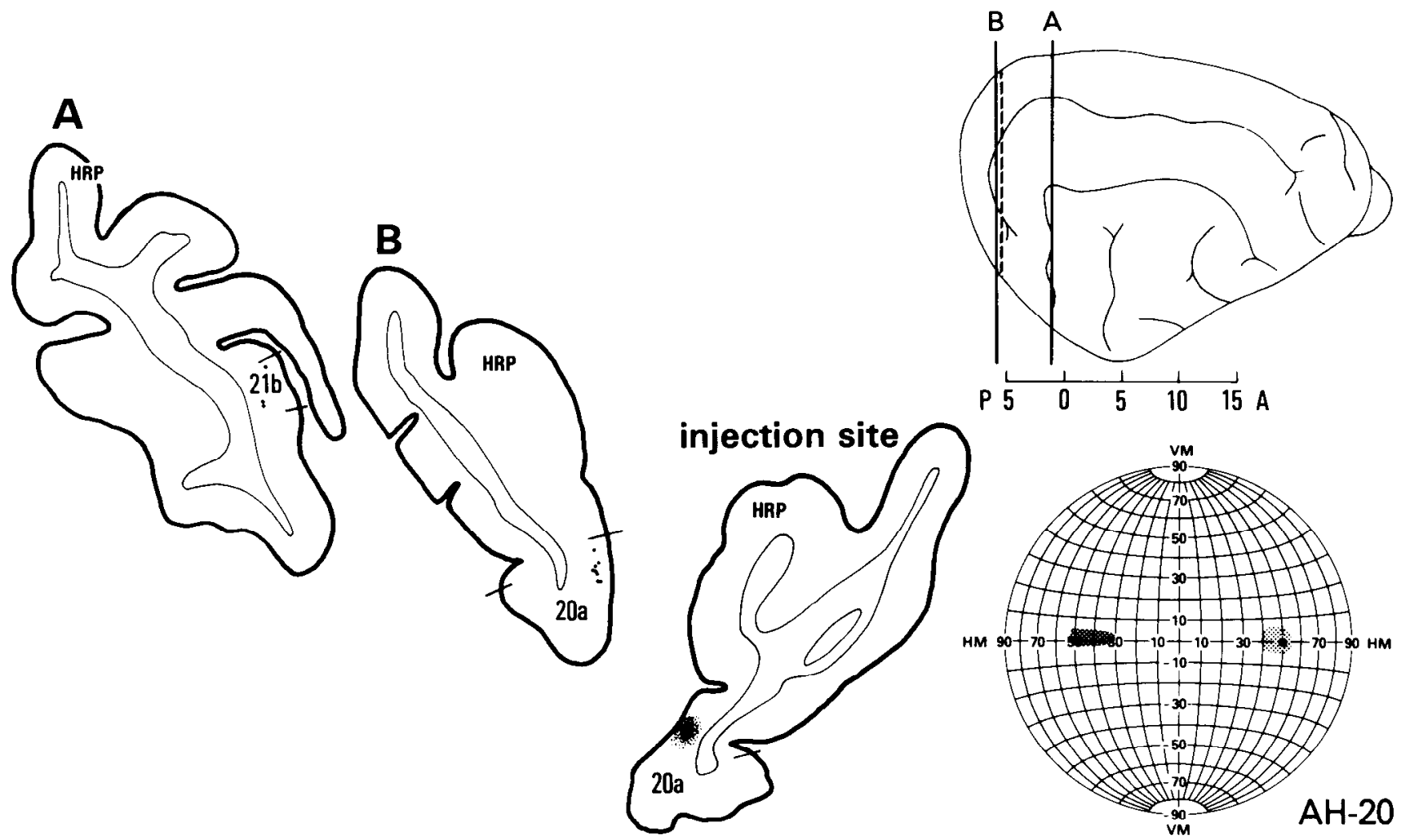

Figure 4. Injection in area $20 \mathrm{a}$. The receptive field center of the injection site was $50,0^{\circ}$ (case AH-20). No [ $\left.{ }^{3} \mathrm{H}\right]$ leucine-labeled terminals were found in this case; see the text for discussion.

called heterotopic areas, and connections between them will be referred to as heterotopic connections. The names of the anteromedial (AMLS), posteromedial (PMLS), ventral (VLS), anterolateral (ALLS), posterolateral (PLLS), and dorsal lateral suprasylvian (DLS) areas of Palmer et al. (1978) are used.

\section{Results}

\section{Homotopic and heterotopic connections}

Label in homotopic cortex. In every experiment, the most prominent HRP and autoradiographic label in the cortex contralateral to the injection site was located in the area that was homotopic to the area of injection. Within homotopic areas, there was a very close correspondence between the position of the injection site in one hemisphere and the positions of labeled neurons and terminals in the opposite hemisphere. For example, in Figure 3 (AH-39), compare the position of label in area 19 in sections $C$ and $D$ to the injection site in the contralateral cortex. In cases where injection sites were located at points close to a representation of the vertical meridian, labeled neurons and terminals in the contralateral homotopic cortex also were found close to the vertical meridian representation (Figs. $2(\mathrm{AH}-23)$ and 5 $(\mathrm{AH}-43))$. However, with the exception of an injection in the peripheral visual field representation in area 17 (AH24, Table I), labeled cells and terminals were in positions symmetrical to the injection site regardless of the receptive field position recorded prior to injection. In the experiment illustrated in Figure 4 (AH-20), a well confined injection was centered at a point in area 20a's visual field representation $50^{\circ}$ from the vertical meridian. The HRP-labeled neurons in contralateral area 20a were restricted to the symmetrical location and were not found at points more ventromedial in area $20 \mathrm{a}$ where central portions of the visual field are represented. Thus, in cases with injections located away from a vertical meridian representation, mirror-symmetrical cortical points representing mirror-symmetrical visual field loci were most heavily interconnected.

Label in heterotopic cortex. In addition to the label in homotopic cortex, in every experiment, we found HRP. labeled cell bodies and $\left[{ }^{3} \mathrm{H}\right]$ leucine-labeled axon terminals in other contralateral visual areas not equivalent to the area of injection (heterotopic connections). The amount of HRP and $\left[{ }^{3} \mathrm{H}\right]$ leucine label in the heterotopic cortex ranged from a small amount in only a few areas, as seen in cases AH-31 and AH-20 (Table I; Fig. 4), to more extensive label in several visual areas, as seen in cases AH-23 and AH-41 (Table I; Figs. 2 and 6). There were many examples of reciprocal as well as many nonreciprocal heterotopic connections (Table I).

The degree to which the amount and distribution of heterotopic label in a single experiment reflected the actual heterotopic connections of the area injected depended upon at least two additional variables. The first variable was the degree of spread of HRP and $\left[{ }^{3} \mathrm{H}\right]$ leucine from the site of injection. Although approximately the same volume of $\mathrm{HRP} /\left[{ }^{3} \mathrm{H}\right]$ leucine mixture was injected in each experiment, the spread of HRP and $\left[{ }^{3} \mathrm{H}\right]$ leucine from the center of the injection varied from experiment to experiment. In cases AH-20 and AH-3 (Table I; Fig. 4), the injections were restricted in size in comparison to 


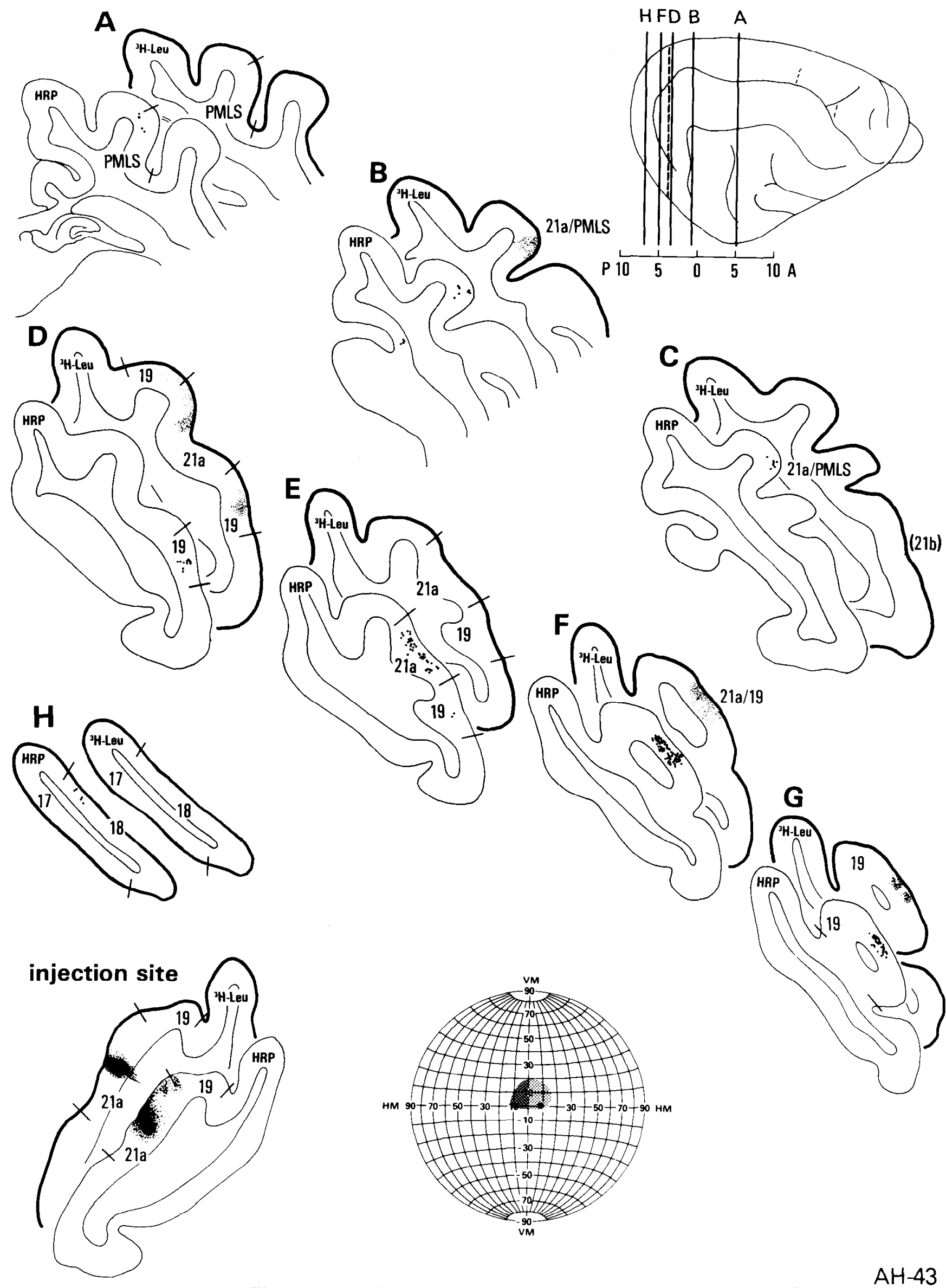

Figure 5. Injection in area 21a. The receptive field center of the injection site was $8,1^{\circ}$ (case AH-43). $\left.{ }^{3} \mathrm{H}\right] \mathrm{Leucine}$ sections $A$, $E$, and $H$ did not contain label. The diagonal line between the names of areas (e.g, 21a/PMLS in $B$ ) signifies that the label was on or very close to a border. The parentheses around $21 \mathrm{~b}$ in $C$ signify uncertainty in assignment of the borders for this area. 

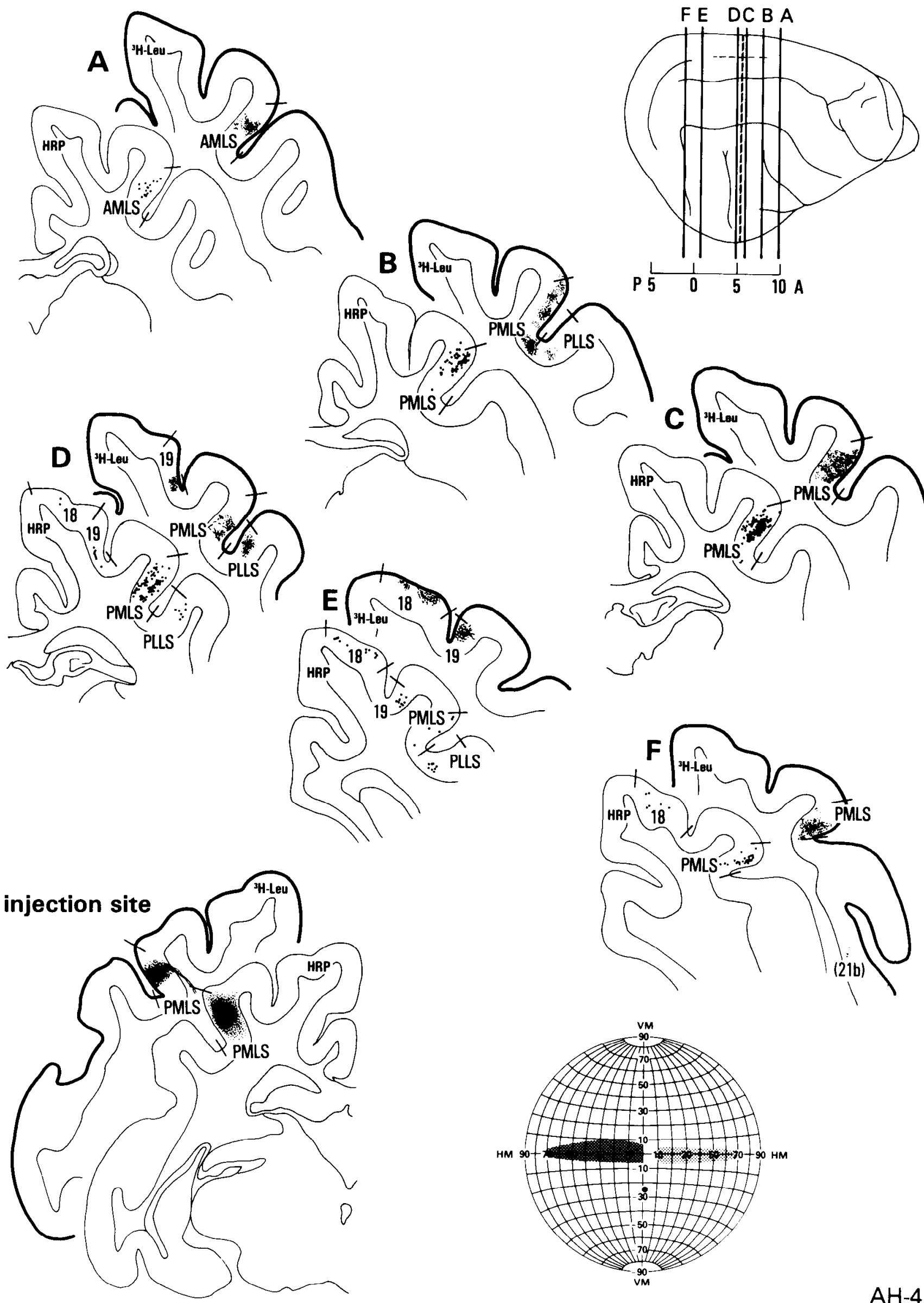

$\mathrm{AH}-41$

Figure 6. Injection in area PMLS. The receptive field center of the injection site was $0,-24^{\circ}$ (case AH-41). The parentheses around $21 \mathrm{~b}$ in $F$ signify uncertainty in assignment of the borders for this area. 


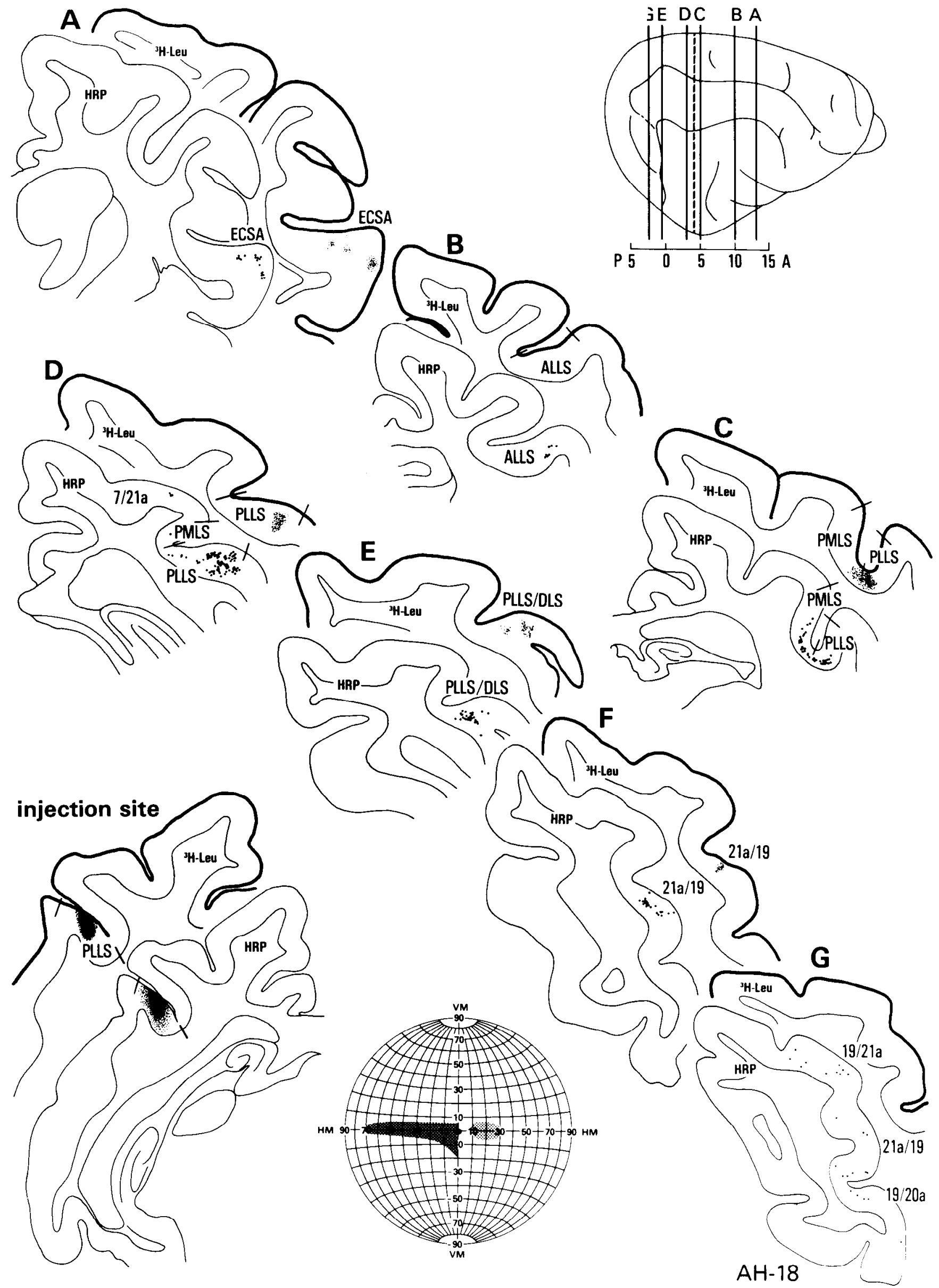

Figure 7. Injection in area PLLS. The receptive field center of the injection site was $0,0^{\circ}$ (case AH-18). $\left[{ }^{3} \mathrm{H}\right] \mathrm{Leucine}$ sections $B$ and $G$ did not contain label. The diagonal line between the names of areas signifies that the label was on or very close to a border. Figure 1 contains photomicrographs of the injection site of this experiment. ECSA, Ectosylvian area. 
most other experiments, and this is probably why there was sparse labeling in these cases. In both experiments, no $\left[{ }^{3} \mathrm{H}\right]$ leucine-labeled terminals were found in either the homotopic or heterotopic cortex. This was believed to be a direct result of the small injection size, since callosal projections to and from areas $20 \mathrm{a}$ and $21 \mathrm{a}$ were demonstrated in other experiments (Table II). The second variable was the position of the injection site within a particular visual field representation. Cases AH-24 and AH-31 exemplify the effect of injection site topography upon the amount of label present in individual experiments. Experiment AH-24 (Table I) is a rather extreme example. An injection was made in area 17 at a location with a receptive field center $74^{\circ}$ from the vertical meridian. This injection was well outside of the zone of origin and termination of callosal fibers in area 17 and, as expected, absolutely no label was found in the contralateral hemisphere. In case AH-31 (Table I), an injection in PMLS was centered $62^{\circ}$ from the vertical meridian. This is a region containing a rather low density of callosal

TABLE II

Summary of callosal connections of 13 retinotopically defined cortical areas

Projections indicated as questionable in Table I are not included. The callosal efferents of an area are obtained by reading across the table. For example, callosal axons whose cell bodies are located in area 17 terminate in areas $17,18,19$, AMLS, PMLS, and 21a. The callosal afferents of an area are obtained by reading down the table. Area 17 receives projections from areas 17, 18, 19, AMLS, and PMLS. The diagonal line that passes from the upper left to the lower right corner passes through blocks that signify homotopic projections. All other blocks signify heterotopic projections. Note that a homotopic projection from area $21 \mathrm{~b}$ is not indicated since we do not have an example of an injection restricted to area $21 \mathrm{~b}$. All of the data on the callosal afferents of area 17, the projection of area 17 to PMLS, and of AMLS to area 18 are based upon the results of an injection along the area 17/18 border (Fig. 2; Table I) and may require subsequent revision.

\section{Origins and destinations of visual callosal axons}

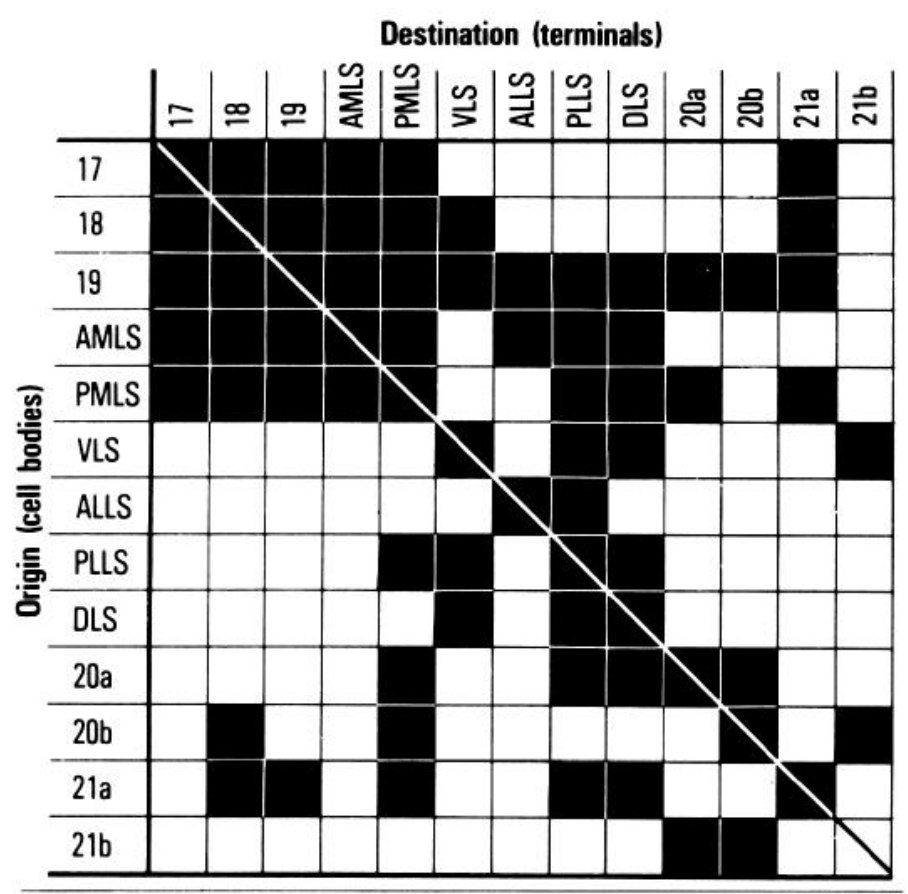

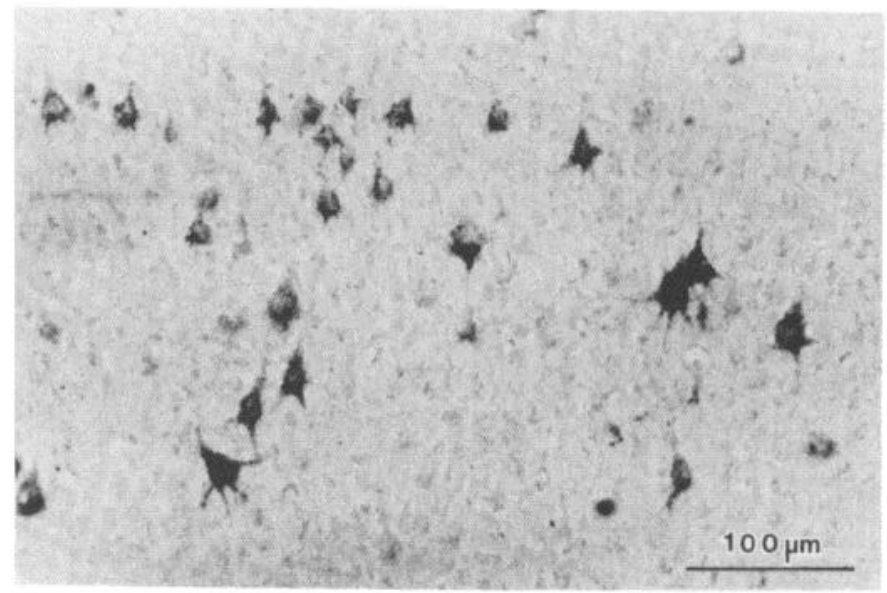

Figure 8. HRP-labeled callosal neurons in area PMLS. This photomicrograph corresponds to the area within the rectangle in Figure 10.

neurons, and although we found both labeled neurons and terminals in the homotopic cortex, heterotopic label consisted of HRP-labeled neurons in a single area (AMLS). Heterotopic label was more widely distributed in experiments with area PMLS injections located closer to the representation of the vertical meridian (Table I, AH-41, AH-6, and AH-11).

In summary, the most prominent callosal connections were between homotopic areas of cat visual cortex, but there was also a very extensive system of heterotopic callosal connections. All of the connections between homotopic areas were reciprocal. Many of the heterotopic callosal connections were reciprocal, but some were not. The homotopic and heterotopic connections demonstrated in all 17 cats were combined to supply the data used to make Table II. This table summarizes the callosal connections of all 13 visual areas. Area 19 appeared to have the most extensive system of callosal efferents. It projected to all retinotopically defined visual areas except area 21b. Areas PMLS and PLLS received more callosal afferents than any other areas, with each receiving projections from different sets of 9 visual areas.

\section{Correspondence of callosal neurons and terminals at individual callosal zones}

Homotopic callosal zones. The reciprocal connections between homotopic areas were very precise and point-topoint in nature. Camera lucida drawings and photomicrographs illustrating the homotopic label in areas 19, PMLS, and ALLS are provided in Figures 8 to 11. In each example, the distributions of HRP-labeled neurons and $\left[{ }^{3} \mathrm{H}\right]$ leucine-labeled terminals correspond very closely. This characteristic was observed in every experiment.

Heterotopic callosal zones. In cases where heterotopic projections were reciprocal, we also found a close correspondence between the positions of HRP-labeled neurons and $\left[{ }^{3} \mathrm{H}\right]$ leucine-labeled terminals. For example, following an injection in area PMLS (AH-41, Fig. 6), both HRP and autoradiographic label were located at similar locations in several heterotopic visual areas. Compare 

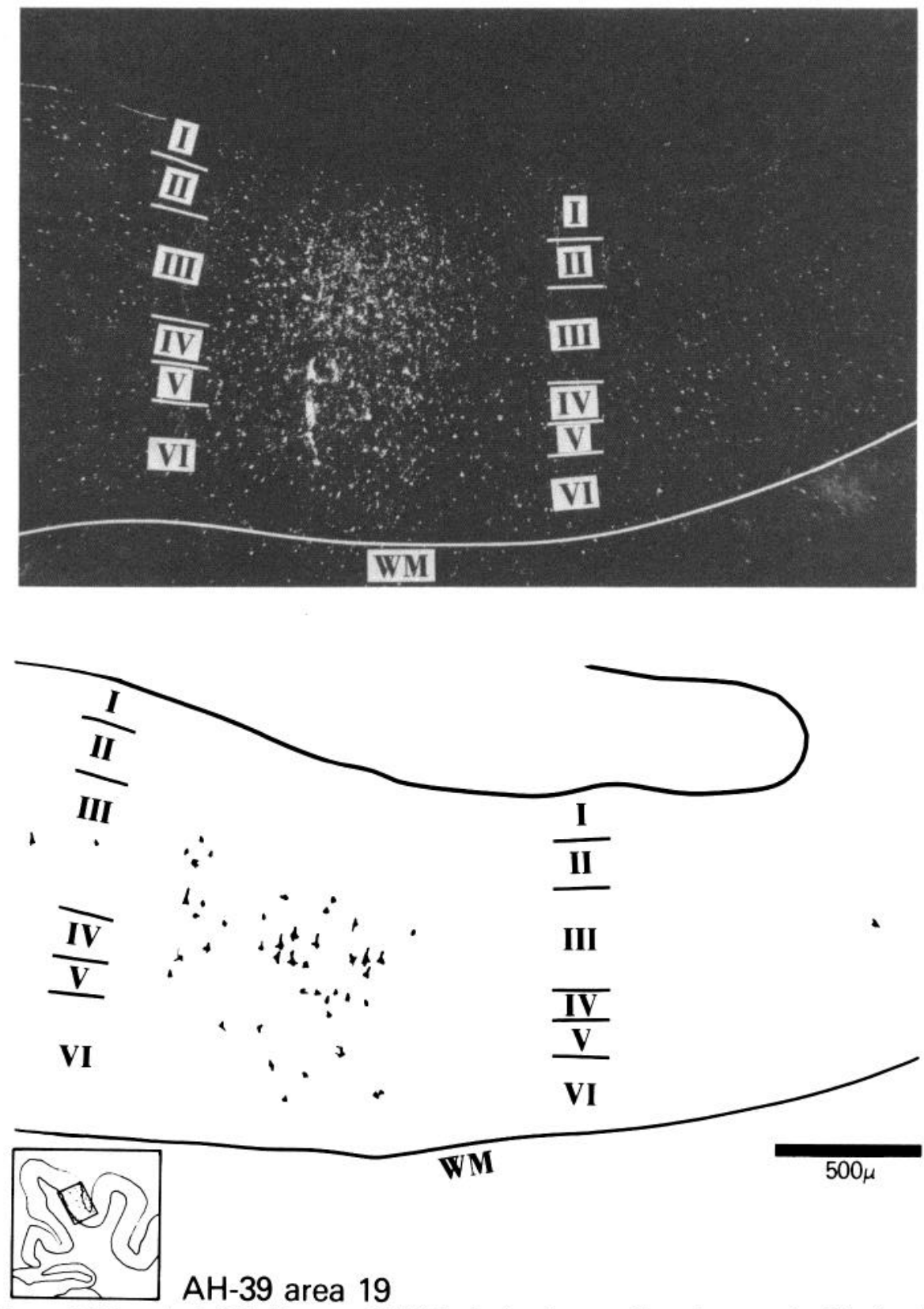

Figure 9. Homotopic label in area 19. This photomicrograph and camera lucida drawing of adjacent sections show the distributions of $\left[{ }^{3} \mathrm{H}\right]$ leucine-labeled terminals and HRPlabeled neurons. WM, White matter.

the HRP and $\left[{ }^{3} \mathrm{H}\right]$ leucine label in areas 19 and PLLS in Figure $6 D$ and in areas 18 and 19 in Figure $6 E$.

\section{Laminar information, cell types, and clustering}

The majority of callosal neurons with homotopic projections were pyramidal cells in lower layer III and upper layer IV. Outside of areas 17 and 18, there was a significant number of pyramidal and fusiform-shaped cells in layers V and VI. The laminar distributions of callosal neurons with homotopic projections in areas 17, 18, 19, AMLS, and PMLS appeared similar to those reported for these same visual areas in the preceding report (Segraves and Rosenquist, 1982). (Compare the laminar distribution of HRP-labeled neurons at the area 17/18 border in Figs. 2 to 8 of the preceding report. For area 19, compare Fig. 9 to the preceding report's Fig. 9. For the lateral suprasylvian visual areas, compare the homotopic label in AMLS (Fig. 13), PMLS (Fig. 10), and ALLS (Fig. 11) to AMLS neurons in Fig. 10 of the preceding report.)

The majority of $\left[{ }^{3} \mathrm{H}\right]$ leucine-labeled terminals were located in layers II, III, and IV (Figs. 9 to 11 and 13). Thus, the laminar positions of labeled callosal terminals coincided with the positions of the majority of labeled callosal neurons in layers III and IV.

Although we did not systematically study the laminar distribution of the neurons and terminals of heterotopic projections, we did observe that the laminar position of a callosal neuron in area PMLS was, to some extent, dependent upon whether it projected to a homotopic or 

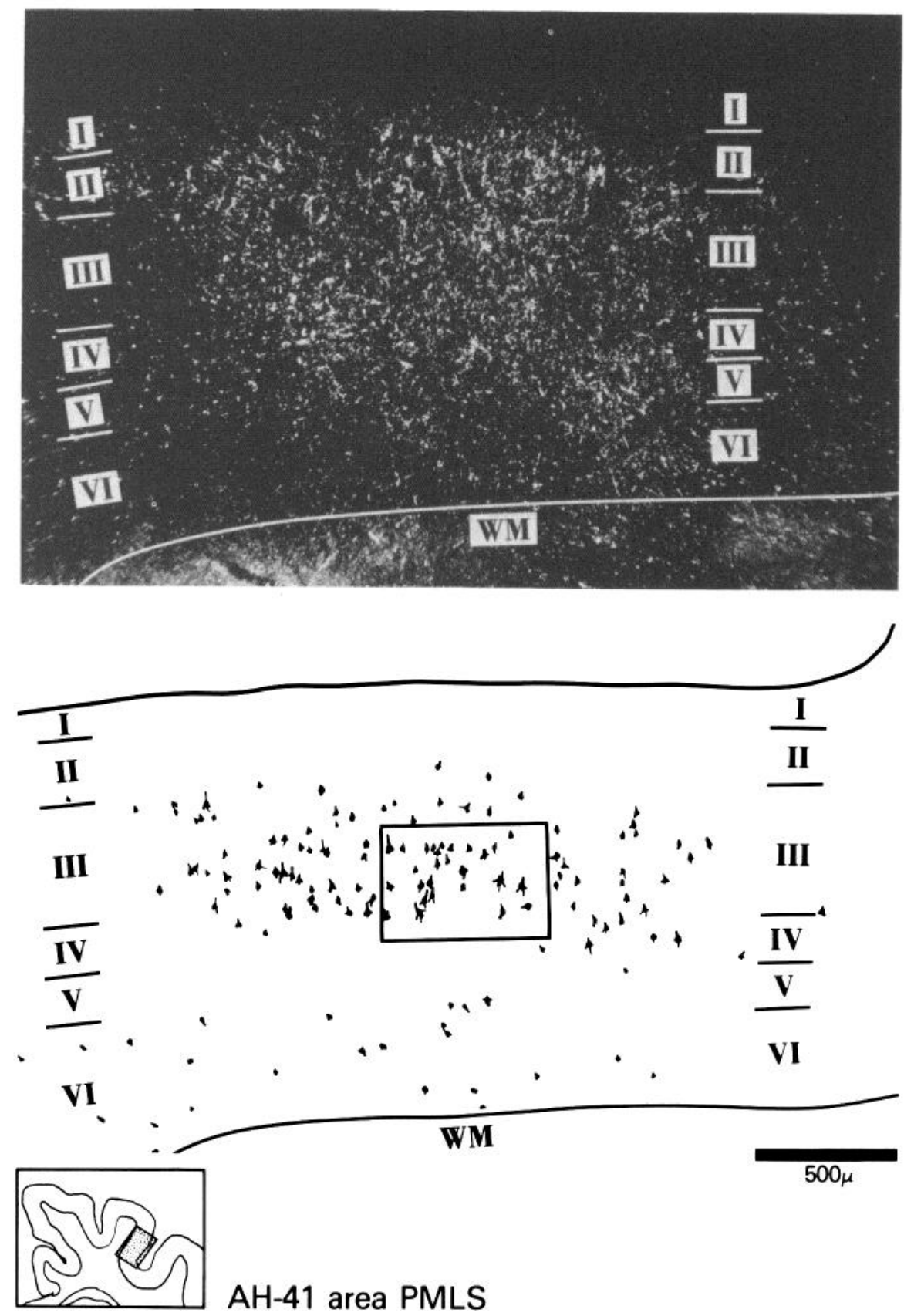

Figure 10. Homotopic label in area PMLS. The area within the rectangle corresponds to the photomicrograph of Figure 8.

heterotopic termination site. As described in our previous report, callosal neurons in PMLS include medium to large pyramids located in deep layer III and upper layer IV and pyramidal and fusiform-shaped cells in layers $\mathrm{V}$ and VI. Cells in PMLS projecting to the contralateral PMLS are located in layers III and IV and, to a lesser extent, in layers V and VI (Fig. 10). However, we found that most PMLS neurons projecting to the contralateral area $17 / 18$ border have their soma restricted to layers $V$ and VI (Fig. 12). This finding also has been reported by Shatz (1977) and by Keller and Innocenti (1981).

In several experiments (AH-21, AH-41, and AH-43, Table I), we found that both HRP-labeled neurons and $\left[{ }^{3} \mathrm{H}\right]$ leucine-labeled terminals in the homotopic cortex were grouped into two to three distinct clusters of label ranging from 600 to $900 \mu \mathrm{m}$ in width (Fig. 13). The HRP and $\left[{ }^{3} \mathrm{H}\right] l e u c i n e$ label in adjacent sections were in equivalent locations. The clusters remained distinct through several adjacent sections; thus, it is unlikely that their presence was due to a fortuitous plane of section or other technical artifact. The center of concentration of both labeled terminals and neurons was in layer III. Clusters were found in only 3 of 17 experiments, and, in each case, they were located at sites of homotopic label. The cortical regions where clusters were found were area AMLS, area PMLS, and the area 19/21a border. 

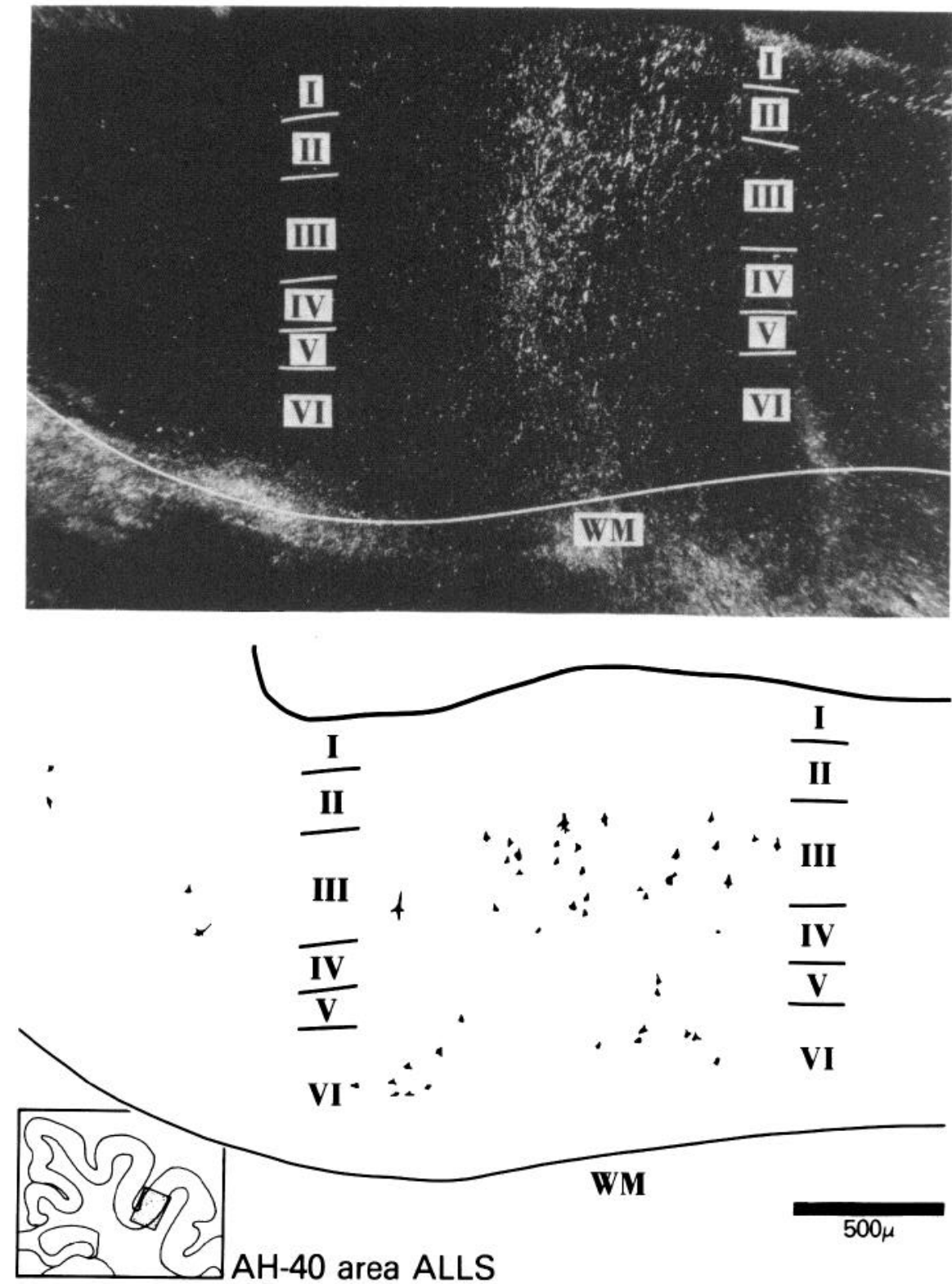

Figure 11. Homotopic label in area ALLS.

\section{Portions of visual field representations that send and/or receive callosal input}

We examined the distribution of homotopic label within visuotopic maps in an effort to extend our observations presented in the previous reported about portions of visual field representations that contain callosal neurons. The important characteristic of these experiments was their ability to show callosal projections to and from small portions of a visual field representation.

As mentioned above, each injection, with the exception of case AH-24, resulted in the transport of label to the contralateral homotopic cortex. The similarity of positions of injection and contralateral homotopic label suggested that symmetrical regions of opposite visual hemifield representations were connected preferentially. Table I lists the receptive field centers of all injection sites. Estimates of the entire area of the visual field representation encompassed by the injection site and area of the visual field representation containing labeled cells in the contralateral homotopic cortex are included in Figures 2 to 7 and 14. An injection on the area 17/18 border (AH-23, Table I) with a receptive field center directly on the vertical meridian produced strong anterograde and retrograde labeling in both areas 17 and 18 (Fig. 2). An area 17 injection with a receptive field center at $74^{\circ}$ azimuth (AH-24, Table I) failed to produce any contralateral labeling. In area 18 , we injected at a site representing $12^{\circ}$ azimuth, resulting in good labeling in the homotopic cortex (AH-44, Table I). In area 19 (AH39, Table I; Fig. 3), an injection with a receptive field center at $20^{\circ}$ azimuth resulted in labeling at the corresponding location in the contralateral cortex. In lateral suprasylvian visual area AMLS (AH-21, Table I), an injection with a receptive field center at $35^{\circ}$ azimuth produced strong homotopic label. Injections in ALLS 


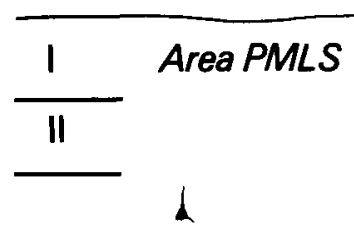

III

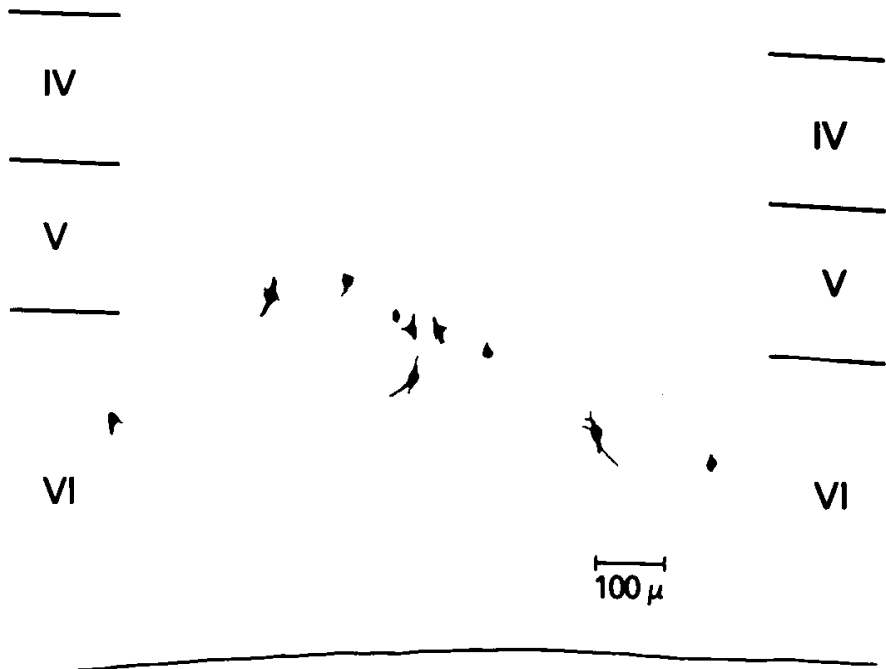

Figure 12. Camera lucida drawing of labeled neurons in lateral suprasylvian visual area PMLS following an injection of HRP at the contralateral area $17 / 18$ border (case AH-23, Table I; Fig. 2). Virtually all PMLS neurons projecting to the contralateral area $17 / 18$ border have their cell bodies located in layers V or VI.

(AH-40, Table I) and VLS (AH-15, Table I) with receptive field centers at $25^{\circ}$ and $23^{\circ}$ azimuth also produced label in the contralateral homotopic cortex. In areas 20a and $20 \mathrm{~b}$ (cases AH-20 and AH-19, Table I; Fig. 4), injections with receptive fields centered at $50^{\circ}$ and $20^{\circ}$ azimuth each produced homotopic label. In area $21 \mathrm{a}$, an injection site with receptive fields centered at $8^{\circ}$ azimuth produced strong homotopic label (case AH-43, Table I; Fig. 5).

Our data for area PMLS are more extensive and are presented in Figure 14. In four experiments, injections were placed in area PMLS at locations with receptive field centers at $0.5^{\circ}, 10^{\circ}, 45^{\circ}$, and $62^{\circ}$ azimuth. In each case, there was a close correspondence between the position of the injection site in the left hemisphere and the location of anterograde and retrograde label in the right hemisphere. The density of label was highest in case AH41 which involved an injection adjacent to the vertical meridian representation. This was consistent with our observation reported in the preceding paper (Segraves and Rosenquist, 1982) that, although callosal neurons are present virtually throughout area PMLS, the density of callosal neurons increases at positions close to the representation of the vertical meridian (see Fig. 6 in the preceding paper). The estimates of the area of the visual field representation encompassed by the injection and the area of the visual field representation containing labeled neurons suggest that the connections between the visual field representations in left and right area PMLS are point-to-point in nature. There does not appear to be any evidence for a convergence of callosal projections to the vertical meridian representation at the fundus of the lateral suprasylvian sulcus, nor is there evidence for diffuse terminations of callosal axons throughout area PMLS.

\section{Callosal connections with cortext outside of the 13 retinotopically defined cortical areas}

Although most labeled neurons and terminals were restricted to the 13 retinotopically defined cortical areas, we sometimes found labeled neurons and terminals in other regions of the cortex. Following injections in areas AMLS and PMLS, we occasionally found labeled terminals at the crown of the middle suprasylvian gyrus (cases AH-21 and AH-6, Table I) in a region corresponding to area 7 of Heath and Jones (1971). Injections in areas ALLS, PLLS, and DLS always labeled cells and terminals in the fundus and ventral bank of the anterior ectosylvian sulcus (cases AH-40, AH-18, and AH-13, Table I; Fig. 7). An injection at the PLLS/DLS border and one in area 21a labeled terminals and cells in a region of posterior suprasylvian cortex ventral to area $21 \mathrm{~b}$ (cases AH-13 and AH-43, Table I) and corresponding to the posterior suprasylvian sulcal area of Heath and Jones (1971). An injection in area 21 a (AH-43, Table I, Fig. $5 B$ ) produced a few labeled neurons at the fundus of the splenial sulcus.

\section{Discussion}

The cat's visual callosal system is a complex array of homotopic and heterotopic connections linking the visual cortex of the two hemispheres. These connections have a highly structured organization that is reflected in the close correspondence of the sites of origin and termination of callosal fibers and in the laminar and possibly even columnar arrangement of callosal cells and terminals. In addition, the visual callosal connections maintain a strictly defined relationship to maps of the visual hemificlds.

Homotopic and heterotopic connections. The homotopic and heterotopic callosal connections of cat visual cortex have been examined by a number of authors (e.g., Hubel and Wiesel, 1965; Garey et al., 1968; Heath and Jones, 1970, 1971; Shatz, 1977; Sanides, 1978a, b; Keller and Innocenti, 1980, 1981). We have extended their findings in a number of ways. By using a small injection of HRP and $\left[{ }^{3} \mathrm{H}\right]$ leucine, we have demonstrated both the afferent and efferent callosal connections of a restricted portion of a visual field representation simultaneously. By recording through the injection pipette, we have determined the retinotopic position of each injection site in the visual field representations of 12 of the 13 retinotopically defined areas of cortex.

We found a very extensive network of homotopic and hererotopic connections (Table II). In each experiment, there was a close overlap in the positions of callosal cells and terminals at homotopic sites (Figs. 2 to 7 and 9 to 11). For heterotopic connections, we found many examples of reciprocal connections, with labeled callosal cells and terminals in close register, and we also found many examples of nonreciprocal projections. A close corre- 

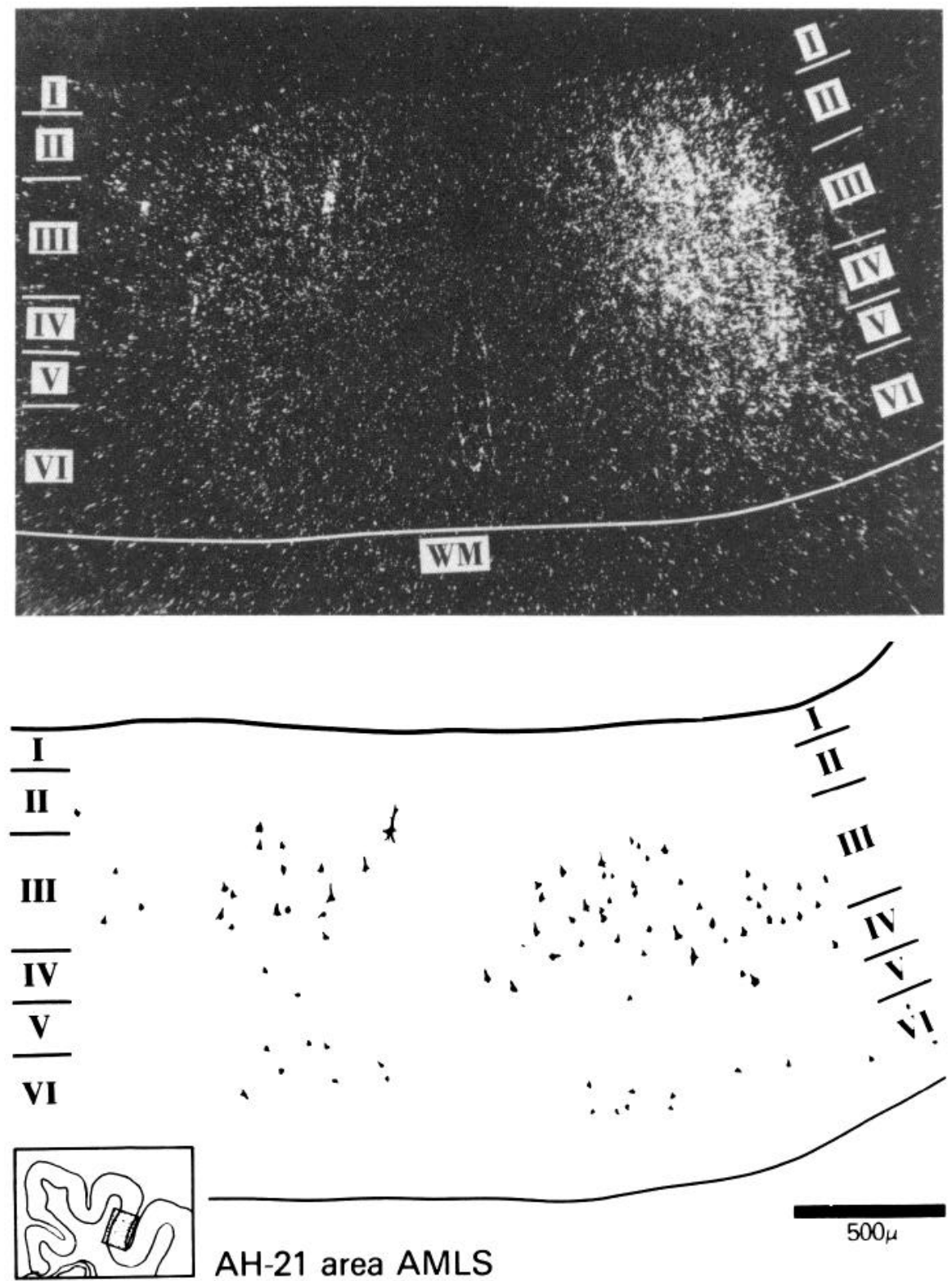

Figure 13. Clustering of callosal neurons and terminals in area AMLS. This photomicrograph and camera lucida drawing of adjacent sections show the distributions of $\left[{ }^{3} \mathrm{H}\right]$ leucine-labeled terminals and HRP-labeled neurons.

spondence in the areal position of callosal cells and terminals also has been seen in the visual cortex of rats and monkeys (Jacobson and Trojanowsky, 1974; Van Essen and Bixby, 1978). Jones et al. (1979) found a precise reciprocal arrangement of homotopic connections in the monkey's somatosensory cortex. However, in contrast to our results for cat visual cortex, they found that the heterotopic projections of somatosensory area I in the monkey were not reciprocal.

Laminar positions. The laminar distributions of callosal neurons that we observed in these experiments were similar to those reported in the preceding paper (Segraves and Rosenquist, 1982). In contrast to a report that callosal neurons in the lateral suprasylvian visual areas with homotopic projections are located almost exclusively in layer III (Keller and Innocenti, 1981), we found that the distribution of cells with homotopic projections was not qualitatively different from the distribution of all callosal neurons demonstrated in the preceding paper. In agreement with Shatz (1977) and Keller and Innocenti (1981), we found that callosal neurons in area PMLS with heterotopic projections to the contralateral area $17 / 18$ border were concentrated primarily in layers $V$ and VI. It is interesting to note that Gilbert and Kelly (1975) found that the projections of the lateral suprasylvian visual areas to ipsilateral areas 17 and 18 also originated primarily from neurons in layer VI. The functional significance of the differences in laminar distributions of cells with homotopic and heterotopic projections in areas PMLS is unknown. The importance of these differences may become clearer as we attain a better understanding of the response properties of the neurons in each layer of this region of the cortex.

In addition to the close overlap of the sites of origin 


\section{injection sites}

PMLS Cases

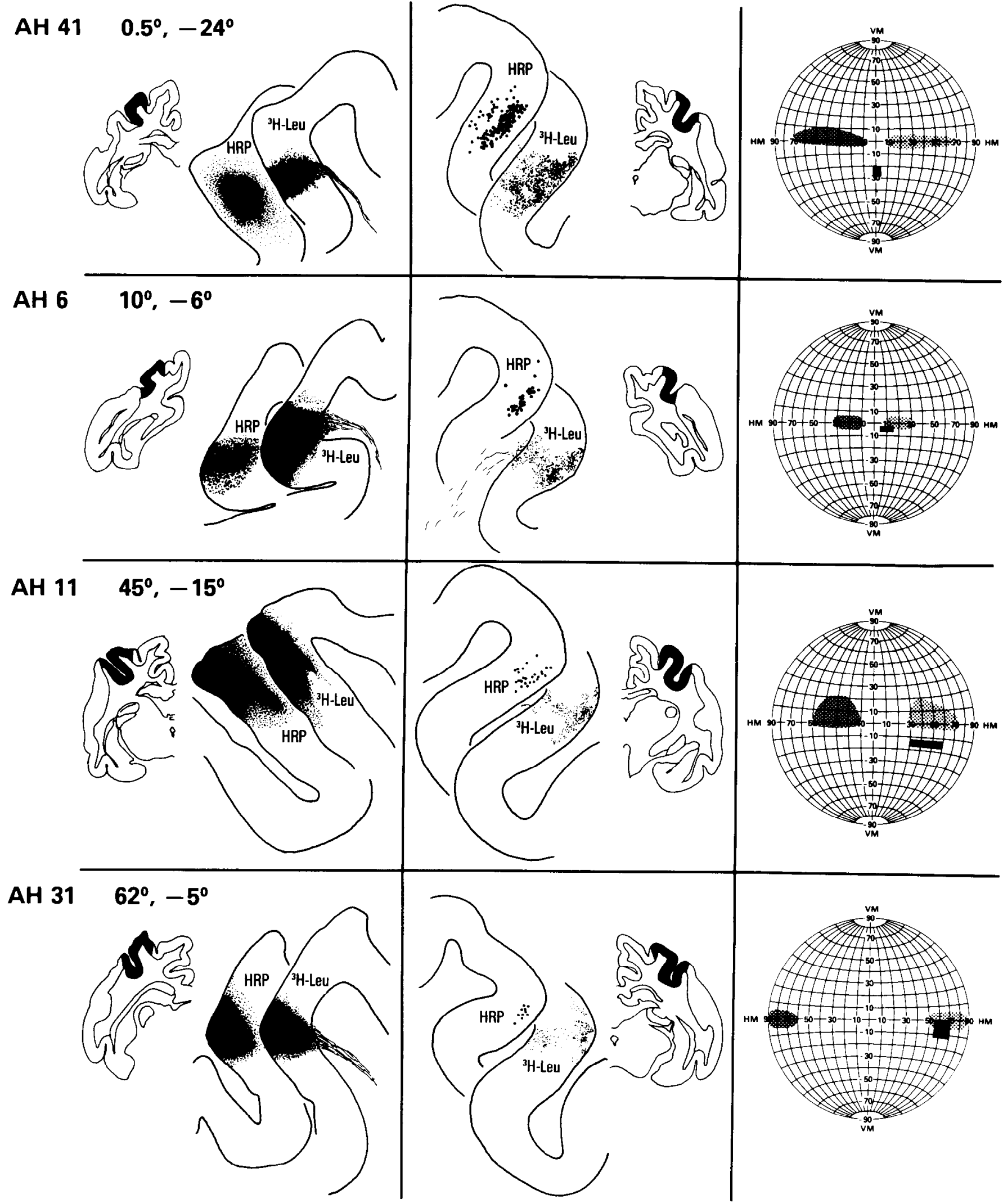

Figure 14. Relationship of injection sites and labeled neurons and terminals to the visuotopic representation in area PMLS. The injection sites and homotopic label in four cases of PMLS injections are shown. In the right hemifield of each globe, the entire receptive field of multiple unit activity recorded at the injection site is blackened. The receptive field center of each injection site is indicated next to the case number. An estimate of the area of the visual field representation encompassed by the injection site is shaded in the right hemifield of the globe. In the left hemifield of the globe, shading covers an estimate of the area of the visual field representation in the contralateral homotopic cortex that contains labeled cells. 
and termination of callosal fibers within individual areas of the cortex, the cells and terminals also occupy similar laminar positions (Figs. 9 to 11 and Shatz, 1977). However, physiological evidence indicates that callosal connections may not be reciprocal at the single cell level, since most callosal neurons do not receive direct synaptic input from the contralateral hemisphere (Harvey, 1980; Innocenti, 1980).

Clustering of callosal neurons and terminals. We found infrequent evidence of a columnar organization of visual callosal neurons and terminals (Fig. 13). Clusters of callosal neurons and terminals were in precise register and were found in homotopic label in areas AMLS and PMLS and at the area 19/21a border. There also has been a report of patches of anterograde and retrograde HRP labeling in the homotopic callosal projections of area 18 (Lamont and Colonier, 1978). Gilbert and Wiesel (1980) have demonstrated a columnar organization of the ipsilateral projections between areas 17 and 19. Using small injections of HRP $(50 \mathrm{nl})$, they demonstrated distinct patches of labeled neurons in areas 17 and 19. Large injections $(0.5 \mu \mathrm{l})$ resulted in continuous bands of label without patches. Based upon Gilbert and Wiesel's (1980) observations, it is possible that we will obtain more frequent demonstrations of columns by reducing the size of our injections.

In comparison to the small amount of evidence for columns in the visual callosal system, evidence for callosal columns in the cat's auditory system and in the somatosensory and motor systems in monkeys is much more substantial (Jones et al., 1975, 1979; Kunzle, 1976; Goldman and Nauta, 1977; Imig and Brugge, 1978; Jenny, 1979). The width of callosal columns or bands in auditory, somatosensory, and motor systems ranges from 500 to $1000 \mu \mathrm{m}$, and, although our data are limited, these dimensions coincide with our measurements of 600 to 900 $\mu \mathrm{m}$ for cat visual callosal columns.

Imig and Brugge (1978) have shown that clusters of callosal neurons and terminals in the cat's auditory cortex coincide with regions where input from the ipsilateral ear makes a significant contribution to neuronal responses. An important achievement in the investigation of visual callosal connections will be to correlate the position of callosal columns with other forms of columnar organization that are based on receptive field properties or on other cortical projection systems.

Relationship of callosal connections to maps of visual space. In the preceding paper (Segraves and Rosenquist, 1982), we noted a progressive increase in the portions of the visual field representation containing callosal neurons in areas 17, 18, and 19 and in the lateral suprasylvian cortex. The results of this study are in agreement with the results of the callosal cut experiments. The use of small injections of a mixture of HRP and $\left[{ }^{3} \mathrm{H}\right] l$ leucine from a recording pipette allowed us to examine the position of callosal terminals as well as neurons. This technique has extended our previous findings by demonstrating the strictly point-to-point nature of callosal connections. We found precisely homotopic connections not only between locations on the vertical meridian representations at the left and right area $17 / 18$ borders but also, for example, between mirror-symmetrical points on the peripheral horizontal meridian representation in left and right PMLS. Evidence for callosal connections between mirror-symmetrical portions of the visual field also has been found in the marmoset (Spatz and Tigges, 1972) and in the owl monkey (Wagor et al., 1975).

A potential problem might arise since our analysis of the relationship between callosal connections and maps of visual space depended upon comparing injection sites, whose retinotopy was known from concurrent recording, with labeled cells and terminals in the contralateral cortex, whose retinopathy was not known directly from recording. In every experiment, homotopic label in the contralateral hemisphere was centered and heaviest at approximately the same anatomical location as the injection site (Figs. 2 to 7 and 14). We believe that the most likely explanation of this is that mirror-symmetrical visual field representations communicate most directly and extensively through the corpus callosum. Another potential problem is that our receptive field recordings focused upon the center of the injection site. We did not attempt to measure directly the degree to which the spread of HRP and $\left[{ }^{3} \mathrm{H}\right]$ leucine involved a larger visual field representation than that indicated by the recording for the center of the injection site. Because of both of these potential difficulties, we have compared both the distribution of homotopic label and the injection sites to standard electrophysiologic maps. This enabled us to estimate the entire area of visual field represented in the homotopic cortex containing labeled neurons and terminals and to estimate the portion of the visual field representation encompassed by the entire injection.

The accuracy of estimates based upon standard maps is dependent upon the amount of variation that exists among cats. Variability from cat to cat has been documented for areas 17, 18, and 19 (Tusa et al., 1978, 1979). Some variability is probably also the case for other visual areas, but this has not yet been documented. Also unknown is the degree to which the two hemispheres of the same animal may vary with respect to the location and retinotopic organization of visual areas. The experiment shown in Figure 6 represents an extreme example of the problem that variability may present. The receptive field center recorded at the injection site was $0.5,-24^{\circ}$. In contrast, our estimates based upon the standard maps (Palmer et al., 1978) place the injection at no closer than $10^{\circ}$ from the vertical meridian and directly upon the horizontal meridian. Despite the possibilities for variability, the receptive field center recorded at each injection site in Figures 2 to 5 is within the boundaries of our estimates based upon the standard maps. There are discrepancies, however, between our recordings and the estimates based upon the standard maps for thie cases shown in Figures 6, 7, and 14.

The traditional assumption is that the corpus callosum connects representations of the vertical meridian and provides a means to join the left and right visual hemifields. This assumption is probably valid for the callosal connections of areas 17 and 18. However, this paper, as well as the preceding report have shown that the callosal neurons of the lateral suprasylvian visual areas and areas 20 and 21 transfer information between cerebral hemispheres that is related to virtually all portions of the visual field. The callosal organization of these areas seems to conflict with the traditional assumption. For 
example, area PMLS receives numerous callosal conncctions throughout most of the visual field representation. Furthermore, at least 9 contralateral cortical areas project to PMLS (Table II). We hypothesize that these callosal afferents to PMLS may be more important for interhemispheric transfer than for fusion of the hemifields. In a behavioral experiment testing pattern discrimination learning and interhemispheric transfer in split chiasm cats, a unilateral lesion to areas AMLS and PMLS produced no learning deficit but did result in poor interhemispheric transfer to the damaged hemisphere (Berlucchi et al., 1979). As noted above, area PMLS receives strong afferent callosal input and this may serve as the substrate for interhemispheric transfer.

There is also electrophysiological evidence for a strong callosal input to area PMLS. Berlucchi (1981) reports that more than $50 \%$ of the cells in area PMLS in split chiasm cats have a portion of their receptive field contributed through the corpus callosum (compared to about $20 \%$ at the area $17 / 18$ border). Receptive fields in PMLS often extend across the vertical meridian into the ipsilateral hemifield (Spear and Baumann, 1975; Palmer et al., 1978; Marzi et al., 1980). Marzi et al. (1980) found binocular fields in PMLS of normal cats that extended up to $30^{\circ}$ into the ipisilateral hemifield. By cutting the corpus callosum during the course of their experiment, Marzi et al. (1980) demonstrated that all of the ipsilateral response through the nasal retina and all but the central $10^{\circ}$ of ipsilateral response through the temporal retina depended on a intact corpus callosum.

In addition to the callosal connections of regions within $30^{\circ}$ of the vertical meridian representation in area PMLS, our anatomical experiments suggest that mirror-symmetrical points in more peripheral portions of the visual field representations are connected (Fig. 14, cases $\mathrm{AH}-11$ and $\mathrm{AH}-31$ ). This conclusion is the result of a comparison of anatomical evidence to electrophysiological maps based upon reccptive ficld centers. However, receptive fields in PMLS are often quite large. Single and multiple unit receptive fields recorded by Palmer et al. (1978) in area PMLS were often $40^{\circ}$ in diameter. Based upon physiological evidence, it has been suggested that the receptive fields of neurons receiving callosal input must include the vertical meridian (Berlucchi, 1981). Recording in split chiasm cats, Berlucchi (1981) reports that, for every binocular cell, the receptive field components contributed by the ipsilateral eye and by the contralateral eye (via the corpus callosum) join at the vertical meridian. Unfortunately, the contribution of callosal inputs originating from nasal retinas, which may include more peripheral receptive fields, are eliminated in split chiasm cats. Whether callosal connections are restricted to cells whose receptive fields include the vertical meridian or not, our anatomical evidence supports the conclusion that regions of the peripheral visual field representation in cat cortex have access to the opposite hemisphere. Thus, stimuli presented in the periphery of the visual field will excite cortical cells in some areas, such as PMLS, that project to the opposite hemisphere.

Despite the precise point-to-point connections that characterize the organization of callosal connections, possibilities for collosally mediated interactions throughout a visual field representation via the intrinsic connections of a cortical area are not excluded. Rizzolatti and $\mathrm{Ca}$ marda (1977) have examined the receptive fields of neurons in area PMLS and have found that a neuron's excitatory response to light within its receptive field can be inhibited by the simultaneous presentation of a second stimulus in virtually any other part of the visual field, including both ipsilateral and contralateral hemifields. Their results raise the possibility that the callosal input to a restricted cortical site is able to influence an entire visual area through the intrinsic connections of that area.

\section{References}

Berlucchi, G. (1981) Recent advances in the analysis of the neural substrates of interhemispheric communication. In Brain Mechanisms and Perceptual Awareness, O. Pompeiano and C. Ajmone Marsan, eds., pp. 133-152, Raven Press, New York.

Berlucchi, G., J. M. Sprague, A. Antonini, and A. Simoni (1979) Learning and interhemispheric transfer of visual pattern discriminations following unilateral suprasylvian lesions in splitchiasm cats. Exp. Brain Res. 34: 551-574.

Cowan, W. M., D. I. Gottlieb, A. E. Hendrickson, J. L. Price, and T. A. Woolsey (1972) The autoradiographic demonstration of axonal connections in the central nervous system. Brain Res. 37: 21-51.

De Olmos, J. S. (1977) An improved HRP method for the study of central nervous connections. Exp. Brain Res. 29: 541-551.

Garey, L. J., E. G. Jones, and T. P. S. Powell (1968) Interrelationships of striate and extrastriate cortex with the primary relay sites of the visual pathway. J. Neurol. Neurosurg. Psychiatry 31: 135-157.

Gilbert, C. D., and J. P. Kelly (1975) The projections of cells in different layers of the cat's visual cortex. J. Comp. Neurol. 163: 81-106.

Gilbert, C. D., and T. N. Wiesel (1980) Interleaving projection bands in cortico-cortical connections. Soc. Neurosci. Abstr. 6: 315 .

Goldman, P. S., and W. J. H. Nauta (1977) Columnar distribution of corticocortical fibers in the frontal association, limbic, and motor cortex of the developing rhesus monkey. Brain Res. 122: 393-414.

Harvey, A. R. (1980) A physiological analysis of subcortical and commissural projections of areas 17 and 18 of the cat. J. Physiol. (Lond.) 302: 507-534.

Heath, C. J., and E. G. Jones (1970) Connections of area 19 and the lateral suprasylvian area of the visual cortex of the cat. Brain Res. 19: 302-305.

Heath, C. J., and E. G. Jones (1971) The anatomical organization of the suprasylvian gyrus of the cat. Ergeb. Anat. Entwicklungsgesch. 45: 4-64.

Hubel, D. H., and T. N. Wiesel (1965) Receptive fields and functional architecture in two nonstriate visual areas (18 \& 19) of the cat. J. Neurophysiol. 28: 229-289.

Imig, T. J., and J. F. Brugge (1978) Sources and terminations of callosal axons related to binaural and frequency maps in primary auditory cortex of the cat. J. Comp. Neurol. 182: 637-660.

Innocenti, G. M. (1980) The primary visual pathway through the corpus callosum: Morphological and functional aspects in the cat. Arch. Ital. Biol. 118: 124-188.

Jacobson, S., and J. Q. Trojanowski (1974) The cells of origin of the corpus callosum in rat, cat and rhesus monkey. Brain Res. 74: 149-155.

Jenny, A. B. (1979) Commissural projections of the cortical hand motor area in monkeys. J. Comp. Neurol. 188: 137-146.

Jones, E. G., H. Burton, and R. Porter (1975) Commissural and cortico-cortical "columns" in the somatic sensory cortex of primates. Science 190: 572-574. 
Jones, E. G., J. D. Coulter, and S. P. Wise (1979) Commisural columns in the sensory-motor cortex of monkeys. J. Comp. Neurol. 188: 113-136.

Keller, G., and G. M. Innocenti (1980) Homotopic and heteroptic callosal connections of visual areas in the cat. In Visual mechanisms in primates and lower mammals: Satellite Symposium of the XXVIII International Congress of Physiological Sciences, July 21-23, 1980, Braunlage, West Germany.

Keller, G., and G. M. Innocenti (1981) Callosal connections of suprasylvian visual areas in the cat. Neuroscience 6: 703-712.

Kunzle, H. (1976) Alternating afferent zones of high and low axon terminal density within the macaque motor cortex. Brain Res. 106: 365-370.

Lamont, P., and M. Colonnier (1978) Cortico-cortical projections to area 18 in the cat: An HRP study. Soc. Neurosci. Abstr. 4: 76.

Marzi, C. A., A. Antonini, M. DiStefano, and C. R. Legg (1980) Callosum-dependent binocular interactions in the lateral suprasylvian area of Siamese cats which lack binocular neurons in areas 17 and 18. Brain Res. 197: 230-235.

Palmer, L. A., A. C. Rosenquist, and R. J. Tusa (1978) The retinotopic organization of lateral suprasylvian visual areas in the cat. J. Comp. Neurol. 177: 237-256.

Rizzolatti, G., and R. Camarda (1977) Influence of the presentation of remote visual stimuli on visual responses of cat area 17 and lateral suprasylvian area. Exp. Brain Res. 29: 107-122.

Sakai, M., H. Sakai, and C. D. Woody (1978) Intracellular staining of cortical neurons by pressure microinjection of HRP and recovery by core biopsy. Exp. Neurol. 58: 138-144.

Sanides, D. (1978a) The retinotopic distribution of visual callosal projections in the suprasylvian visual areas compared to the classical visual areas $(17,18,19)$ in the cat. Exp. Brain Res. 33: 435-444.

Sanides, D. (1978b) The visuotopic distribution of the callosal projection in the cat. Neurosci. Lett. (Suppl.) 1: S382.

Segraves, M. A. (1979) Interhemispheric connections of retino- topically defined visual cortical areas in the cat. Soc. Neurosci. Abstr. 5: 807.

Segraves, M. A., and A. C. Rosenquist (1980) The location of callosal projection neurons in relationship to maps of the visual field in cat cortex. Soc. Neurosci. Abstr. 6: 673.

Segraves, M. A., and A. C. Rosenquist (1982) The distribution of the cells of origin of callosal projections in cat visual cortex. J. Neurosci. 2: 1079-1089.

Shatz, C. J. (1977) Anatomy of interhemispheric connections in the visual system of Boston Siamese and ordinary cats. J. Comp. Neurol. 173: 497-518.

Spatz, W. B., and J. Tigges (1972) Experimental-anatomical studies on the "middle temporal visual area (MT)" in primates: I. Efferent cortico-cortical connections in the marmoset Callithrix jacchus. J. Comp. Neurol. 146: 451-464.

Spear, P. D., and T. P. Baumann (1975) Receptive field characteristics of single neurons in lateral suprasylvian visual area of the cat. J. Neurophysiol. 38: 1403-1420.

Tusa, R. J., L. A. Palmer, and A. C. Rosenquist (1978) The retinotopic organization of area 17 (striate cortex) in the cat. J. Comp. Neurol. 177: 213-236.

Tusa, R. J., A. C. Rosenquist, and L. A. Palmer (1979) Retinotopic organization of areas 18 and 19. J. Comp. Neurol. 185: 657-678.

Tusa, R. J., L. A. Palmer, and A. C. Rosenquist (1981) Multiple cortical visual areas: Visual field topography in the cat. In Cortical Sensory Organization, Vol. 2, C. N. Woolsey, ed., pp. 1-31, Humana Press, Clifton, NJ.

Van Essen, D. C., and J. L. Bixby (1978) The distribution of cells projecting interhemispherically in extrastriate visual cortex of the macaque. Soc. Neurosci. Abstr. 4: 649.

Wagor, E., C. S. Lin, and J. H. Kaas (1975) Some cortical projections of the dorsomedial visual area (DM) of association cortex in the owl monkey, Aotus trivirgatus. J. Comp. Neurol. 163: 227-250. 